\title{
Author Index Vol. 36, 1997
}

Alleva, E. 65 Aloe, L. 65 Ampollini, P. 1,25 Anderer, P. 117, 130 Angelucci, F. 65 Aschauer, H.N. 69 Ashby, C.R., Jr. 96

Babej-Dölle, R.M. 73 Bandelow, B. 182 Bartmann, U. 182 Bauer, U. 100 Baumgart-Schmitt, R. 194 Bernardi, F. 37 Bersani, G. 65 Bes, F. 194,211 Bocchetta, A. 37 Born, J. 87 Bovier, P. 164 Bracha, H.S. 22 Brandstätter, N. 117, 130 Broderick, A. 57 Broocks, A. 182

Campana, A. 13 Carlen, P.L. 53 Carter, J.G. 32 Catapano, F. 8 Chiaramonti, R. 153 Chiodera, P. 1 Chollet, D. 164 Coenen, A. 42 Coiro, V. 1 Crabtree, B.L. 32

Deckmann, M. 172 Dierks, T. 100 Dorn, H. 100

Eilers, R. 194

Fallgatter, A.J. 153 Fehm, H.L. 87 Fein, G. 83 Feraro, R. 22, 172 Frey, R. 117,130 Furac, I. 62

Gallhofer, B. 100 Gambini, O. 13 Gardner,E.L. 96 George, A. 182 Gifford, A.N. 96 Gruber, G. 117,130 Grünberger, J. 117,130 Gutman, J. 172

Hadler, D. 73 Handzel, Z.T. 172 Hartmann, W. 57 Hatzinger, R. 69 Hegerl, U. 100

Herrmann, W.M. 73, 100, 194 Hilleret, H. 164 Hillmer-Vogel,,U. 182 Hofmann, S. 73 Hornik, K. 69 Huang,|Y.-H. 177

Iannitelli, A. 65 Ihl, R. 100 Ishihara, H. 112 Ivezic, S. 62

Jakovljevic, M. 19,62 Jann, M.W. 32

Kimhi, R. 22,172 Klösch, G. 117,130 Kudo, T. 112 Kudoh, A. 112 Kunz, D. 211

Lam, Y.W, F. 32 Langer, G. 69 LaRocca, A. 159 Lemke, M.R. 57 Lenzinger, E. 69 Levine, J. 22, 172 Levy, P. 172 Leykin, I. 172 Linzmayer, L. 117,130

Loviselli, A. 37 Luijtelaar, G. van 42 Lykouras, L. 5 
Macciardi, F. 13 Maes, M. 107 Maggini, C. 25 Maj, M. 8,159 Malafosse, A. 164 Mandl, M. 117,130 Manni, L. 65 Marchesi, C. 1,25 Mariotti, S. 37 Martine, T. 22 Maselli, P. 65 Matsuki, A. 112 Maurer, K. 100 Meszaros, K. 69 Meyer, T.F. 182 Mölle, M. 87 Monteleone, P. 8, 159 Mossa, P. 37 Mouthon, D. 164 Mück-Šeler, D. 19 Müller, ThJ. 153 Muscas, G.C. 153

Natale, M. 159 Noldy, N, E. 53

Oruc, L. 62

Paganini, M. 153 Papageorgiou, G. 5 Papasteriades, C. 5 Pappa, H. 5 Pèzzola, A. 49 Pietrowsky, R. 87 Pitts, W.M. 32 Pivac, N. 19 Popoli, P. 49

Raeymaekers, P. 62 Raffin, Y. 164 Reggio, R. 49 Rudolf, S. 87 Rüther, E. 182

Sakalis, G. 5 Salamy, A. 83 Saletu, B. 117,130 
Saletu-Zyhlarz, G. 117,130 Salfi, M. 83 Scarone, S. 13 Schönbeck, G. 69 Shinitzky, M. 172 Sieghart, W. 69 Signifredi, R. 25 Sim, C.-B. 177 Strik, W.K. 153 Takeuchi, Y. 188 Theodoropoulou, St. 5 Thompson, P. 107 Tortorella, A. 8 Tsai, S.-J. 177

Ueda, S. 188 Unrug, A. 42

Van Broeckhoven, C. 62 Velluzzi, F. 37 Verheyen, G.R. 62 Versari, A. 153 Volpi, R. 1

Wang, Y.-C. 177 Weyer, G. 73 Widmer, J. 164 Winterer, G. 100

Yang, Y.-C. 177 Yoshida, T. 188 Yoshimoto, K. 188 Yu, M.-F. 177

Zeitelberger, M. 57 Zompo, M. del 37

\section{KAIIGEK}

E-Mail karger@karger.ch Fax+41613061234 http://www.karger.ch 
